\title{
mHealth medication and blood pressure self-management program in Hispanic hypertensives: a proof of concept trial
}

This article was published in the following Dove Press journal:

Smart Homecare Technology and TeleHealth

3 October 2013

Number of times this article has been viewed

\author{
John C Sieverdes' \\ Mathew Gregoski' \\ Sachin Patel' \\ Deborah Williamson' \\ Brenda Brunner-Jackson' \\ Judith Rundbaken' \\ Eveline Treiber' \\ Lydia Davidson' \\ Frank A Treiber ${ }^{1,2}$ \\ 'Technology Applications Center \\ for Healthful Lifestyles, College \\ of Nursing, ${ }^{2}$ College of Medicine, \\ Medical University of South Carolina, \\ Charleston, SC, USA
}

\begin{abstract}
Patient nonadherence to medication regimens and provider therapeutic inertia (failure to respond in timely manner to clinical data) are two primary contributors to ineffective chronic disease management. This 3-month proof of concept trial used an iterative design approach guided by self-determination theory and the technology acceptance model to develop a culturally sensitive, patient-centered, and provider-centered mobile health medication and blood pressure self-management program. Cellular connected electronic medication trays provided reminder signals for patients to take medications and smartphone messaging reminded patients to take at-home blood pressures using a Bluetooth-enabled monitor. Providers were given bimonthly feedback. Motivational and reinforcement text and audio messages were sent based upon medication adherence rates and blood pressure levels. Ten Hispanics with uncontrolled essential hypertension were randomized to standard care and Smartphone Medication Adherence Stops Hypertension (SMASH) intervention groups. Primary outcomes of provider and patient acceptability of the program were found to be high. Retention rates for the 3-month program were $100 \%$, with mean \pm standard deviation overall medication adherence for the SMASH group at $97.2 \% \pm 2.8 \%$, with all strongly believing the program helped them remember to take their medication. SMASH participants measured their blood pressure every 3 days $83.2 \% \pm 6.0 \%$ of the time and completed $89.2 \% \pm 19.06 \%$ of the expected readings. Nonparametric tests showed statistical significance for resting blood pressure changes between groups at months $2(P=0.016)$ and $3(P=0.008)$, with a pre-intervention to 3 -month mean systolic blood pressure reduction of $47.2 \mathrm{mmHg}$ for the SMASH group compared with a reduction of $12 \mathrm{mmHg}$ for the standard care group. Change in scores between pre-intervention and months 1, 2, and 3 for ambulatory blood pressure values found that the SMASH group exhibited consistently greater reductions for 24-hour, wake, and sleep categories compared with little change in the standard care group, although statistical significance was not reached. Principles of patient-centered care highlight partnering with intended users and implementers are important when developing a culturally sensitive intervention that is meaningful and effective.
\end{abstract}

Keywords: medication adherence, clinical inertia, self-determination theory, mHealth, blood pressure control, essential hypertension, Hispanics

\section{Introduction}

Essential hypertension is the most commonly diagnosed chronic disease in the US, affects one third of adults, and is an independent risk factor for stroke, renal failure, congestive heart failure, and cardiovascular events. ${ }^{1}$ Despite improvements in awareness, treatment, and control of essential hypertension, significant ethnic and racial disparities persist. Hispanics, the largest and fastest growing minority group in the US, have the highest rates of uncontrolled essential hypertension, the lowest rates 
of consistent primary medical care, and the highest rate of hypertension-related mortality. ${ }^{2-4}$

Two leading factors responsible for uncontrolled essential hypertension are medication nonadherence and therapeutic inertia. ${ }^{5-8}$ Medication adherence refers to whether patients take their medications at the correct times and dosages as prescribed by their physicians. ${ }^{9}$ Sustained adherence to medication regimens for essential hypertension has been shown to control blood pressure (BP) and reduce cardiovascular disease-related events. ${ }^{10,11}$ For example, using medication possession ratios, hypertensives who exhibited high medication adherence $(80 \%-100 \%)$ were $45 \%$ more likely to achieve BP control. ${ }^{12}$ Despite this demonstrated efficacy, medication nonadherence is the leading modifiable barrier to BP control. ${ }^{6,7}$ More than $50 \%$ of new patients with essential hypertension stop taking their prescribed medications within the first year after diagnosis, and less than $65 \%$ remain in active care after 3 years. ${ }^{13}$ Perez reviewed the literature on self-management of essential hypertension among Hispanics and identified the primary barriers, which included poor planning, forgetfulness, health care access, communication barriers, and affordability. ${ }^{14}$

The other leading factor, therapeutic inertia, is defined as the provider's inaction to modify medical regimens (eg, dose titration, start new medications) when treatment goals are not attained. Therapeutic inertia may explain why patients remain hypertensive despite having high adherence to medical regimens. ${ }^{8} 15$ Absence of data regarding patients' at-home BP and providers' nonrecognition of best practice guidelines both contribute to this problem. ${ }^{8,16,17}$ A study that followed 7,253 patients with uncontrolled essential hypertension in the Southeastern US found that, despite having in-clinic hypertensive BP values, medication adjustment occurred only $13.1 \%$ of the time. ${ }^{8}$

\section{Status of trials on self-management of essential hypertension and clinical inertia}

Recent reviews of a collective total of 133 randomized controlled trials (37 RCTs; $; 78$ RCTs; ${ }^{18} 18 \mathrm{RCTs}^{19}$ ) involving patients with uncontrolled essential hypertension concluded that BP self-monitoring (readings brought/mailed/ phoned into clinics), medication reminder tactics (live or automated phone reminders), and/or in combination with individual counseling, often improve adherence, reduce therapeutic inertia, and result in small but significant declines in BP. However, only $40 \%-50 \%$ of participants reached BP control (eg, clinic BP $<140 / 90 \mathrm{mmHg}$ ) and improvements often deteriorated following intervention cessation. . $^{5,18,19}$
Several recent mobile health (mHealth) feasibility trials that have been done in patients with essential hypertension incorporated BP self-monitoring using Bluetooth-enabled devices and provider BP summary reports. Compared with standard care groups, mHealth groups showed significant BP reductions, although medical standards for BP control (BP $<140 / 90 \mathrm{mmHg}$ ) were not typically achieved. ${ }^{20}$ Lai conducted a quasi-experimental 9-month study in Hispanics with uncontrolled essential hypertension involving BP selfmonitoring and face-to-face pharmacist-delivered educational consultations. ${ }^{21} \mathrm{BP}$ control was reached by 2 months (reduced from $150 / 95 \mathrm{mmHg}$ to $133 / 83 \mathrm{mmHg}$ ) and was maintained throughout the study.

To our knowledge, no published study has incorporated a combination of mHealth technology-based medication intake and BP self-monitoring tactics with Hispanics, the underserved minority group with the highest rate of uncontrolled essential hypertension. ${ }^{2-4}$ Our research has confirmed other findings that Hispanics have high utilization rates of smartphones and are highly receptive to use health technology to improve adherence to medical care regimens and communicate with health care providers. ${ }^{22,23}$ Before largescale trials can test the efficacy of this idea, smaller trials need to be performed to assess the acceptability of such an approach. Therefore, we conducted a 3-month proof of concept RCT involving Hispanics with uncontrolled essential hypertension. The primary purpose was to test the feasibility of a real-time medication reminder and BP self-monitoring system in conjunction with behavioral theory strategies to improve BP self-management, with the secondary purpose of investigating signals of clinical changes in resting and ambulatory BP.

\section{Materials and methods Participants}

Potentially eligible patients were identified via medical records from two federally qualified health center practice sites that provide health care for individuals predominantly of lower socioeconomic status and living in rural, underserved areas within Charleston County, South Carolina. Inclusion criteria were: Hispanic or Latino ethnicity, diagnosed with essential hypertension, prescribed medication(s) for essential hypertension, and systolic BP $>140 \mathrm{mmHg}$ on last clinic visit within the previous 12 months. Exclusion criteria were: $<21$ years of age, inability to self-administer medications, measure own BP or use a smartphone, a history of psychiatric illness or substance abuse, medical diagnoses besides type 2 diabetes mellitus (eg, cancer treatment within previous 2 years, 
stroke, myocardial infarction, Parkinson's disease), pregnant, lactating, or intention of becoming pregnant during the trial, participating in another study, and inability to speak, hear, or understand English or Spanish. Patients who met the initial eligibility criteria were contacted by phone using Spanishspeaking study staff and, if interested, scheduled for a clinic BP screening. The Medical University of South Carolina internal review board approved the study protocol.

\section{Physiologic measurements}

Clinic-based anthropometric and resting BP evaluations were conducted prior to randomization (ie, pre-intervention) and again at months 1,2, and 3. Patients were seated upright with their right arm resting on a table at heart level. Proper cuff size was applied on the right arm for a Dinamap ProCare 200 (GE Healthcare, Buckinghamshire, UK) at each evaluation. The Dinamap has been validated using standard auscultatory methods from the British Hypertension Society and the Association for Advancement of Medical Instrumentation (AAMI) in adults. ${ }^{24}$ The Dinamap, as well as the other BP devices used in the study, were calibrated following the manufacturer's specifications. Readings were immediately taken, again after a 5-minute rest, and two additional readings were taken separated by 2-minute intervals. The average of the last two readings was used in the analyses for initial eligibility and BP evaluations. Essential hypertension was defined as a systolic BP $\geq 140 \mathrm{mmHg}$ using Joint National Committee (JNC) guidelines. Systolic $\mathrm{BP}$ was used as the BP control selection parameter since controlling systolic BP results in commensurate diastolic BP control for a majority of patients. ${ }^{1}$ In addition, height, weight, and waist circumference were taken using standard measurement protocols. ${ }^{25,26}$

\section{Ambulatory BP evaluations}

The 24-hour ambulatory BP was assessed pre-intervention and at months 1,2, and 3. Measurements were automated to record BP readings every 20 minutes during daytime hours, and every 30 minutes during night-time hours using 90207 monitors (SpaceLabs Inc., Issaquah, WA, USA). The instrument has been validated following the British Hypertension Society and AAMI protocols using standard auscultatory readings. ${ }^{27}$ Acceptability of ambulatory readings was based on established criteria. ${ }^{28,29}$ Averages for all readings were created within designated categories of 24 hours, wake (ie, 8 am to $10 \mathrm{pm}$ ), and sleep (ie, 12 am to $6 \mathrm{am}$ ). For each participant, a minimum of at least $50 \%$ of the expected number of readings was required for each time category, otherwise that category was considered missing.

\section{MedMinder device}

The MedMinder (model: Maya, MedMinder Inc., Newton, MA, USA) (Figure 1) medication tray uses an $110 \mathrm{~V}$ power source, has 28 compartments (up to four doses daily $\times 7$ days), and provides reminder signals through a cellular network to a Health Insurance Portability and Accountability Act compliant server. When the medication is to be taken, a blinking light from a specific dose compartment activates. If the pill container is not opened, removed, and returned in 30 minutes, a loud chime also activates for 30 minutes after which an automated personalized reminder message is sent via phone call or text message. The medication tray dates and time stamps each sequence when one of the compartments is removed and sends the information wirelessly to the cellular network in real time. Failure to open the compartment after 90 minutes triggered an automated email message to the study coordinator.

\section{Theory-based behavior change}

Self-determination theory was used as the underlying behavioral change theory for this mHealth program. Selfdetermination theory is directed at building confidence (ie, self-efficacy) in the ability to engage in and increase motivation to sustain desired behaviors. Building sustained motivation in self-determination theory focuses upon development of autonomous regulation, which is fostered by inculcating a sense of ownership and meaning over one's behavior change. ${ }^{30,31}$ Behaviors consistent with personal values and/or short-term and long-term life goals are more likely to be sustained than those resulting from external cues (eg, “doctor's orders") or negative internal (eg, shame, guilt) pressures. ${ }^{31}$ Based upon their disease management behaviors

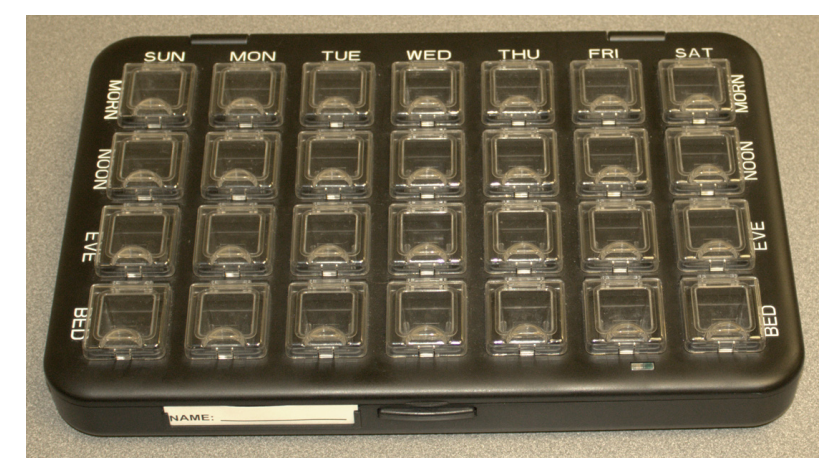

Figure I MedMinder medication tray. Note: Maya, MedMinder Inc., Newton, MA, USA. 
(ie, medication adherence, BP monitoring), patients received personalized feedback and motivational messages (see Table 1 for a detailed explanation).

\section{Randomization process and intervention overview}

Following the pre-intervention screening evaluations and informed consent, patients were randomized to either standard care control or the Smartphone Medication Adherence Stops Hypertension (SMASH) intervention groups. SMASHers received a Motorola Droid 2 or X (Motorola Inc., Schaumburg, IL, USA) with an application installed to automatically acquire and forward encrypted home-based BPs from their Bluetoothed BP device (A\&D model UA-767PlusBT San Jose, CA, USA) to a secure server. The A\&D monitor was calibrated and satisfied the British Hypertension Society and AAMI criteria for systolic and diastolic BP accuracy compared with standard auscultatory readings. ${ }^{32}$ SMASHers were requested to record their BP in the morning and evening every 3 days using the same protocol as mentioned previously for the clinic BP measurements. They also received a MedMinder electronic medication tray and were individually instructed on how to operate all devices and successfully demonstrated

Table I Tailored motivational messages for SMASH group example

Example of participant in SMASH intervention arm

Participant three is a 25 -year-old unmarried male with $\mathrm{EH}$ and noninsulin-dependent type 2 diabetes (T2D). His family history includes a mother with EH, T2D, and chronic kidney disease (CKD) who recently received a kidney transplant, a father who suffers from $\mathrm{EH}, \mathrm{T2D}$, and CKD and receives dialysis, a brother who lost a foot due to T2D complications, and a sister who has T2D and EH

Personal values: strong religious beliefs, eg, following God's word, one's body is God's temple

Short-term goals: increasing energy levels, to spend more time in music ministry, playing with children, engaging in soccer

Long-term goals: becoming healthier for marriage, able to raise children, watching children grow up, not repeating family history of premature disability

Condition: medication doses taken correctly over 24-hour period

"Name, you are doing so well. You took your medication correctly today!"

"You are making your body strong, healthy, and ready to enjoy God's future blessings."

Condition: medication taken but not at correct time

"Taking medicine is good, taking it at the prescribed time is better!

Try today. Your family history does not have to be your future!"

Condition: missed medication doses over 24 hours

"Oops, looks like the last day or so have been really busy for you!

Try and remember to take your medicine every day! God made

you, and you are taking care of His gift of life!"

Abbreviations: SMASH, Smartphone Medication Adherence Stops Hypertension; $\mathrm{EH}$, essential hypertension. their use before beginning the study. A SMASH navigator (ie, research staff) and the SMASHer set the times that medications would be taken daily. All devices securely relayed all information to our teleprocessing center. SMASHers received written and oral information on adherence criteria: take medications within 90 minutes before or after designated times (ie, 3-hour window) and take resting BP using the supplied A\&D monitor in the morning and evening every 3 days.

In preliminary interviews, we found that God, family, and naturalistic healers (curanderos) played important roles in Hispanics' health care-related behaviors. Thus, a bilingual research technician interviewed SMASHers to determine what their primary interests were and included life values, short-term and long-term goals, roles of cultural values (eg, familismo, machismo), and beliefs (eg, religiosity, curanderos) on health care behaviors. This information was used to generate personalized motivational and feedback messages along with other generic motivational and reinforcement messages (see Table 1 for examples). They received these messages based upon their medication adherence levels from the previous day during the first month, and then every several days afterwards via their preferred mode of delivery (text, email, voice mail). These messages were not automated during the proof of concept study. They did receive immediate smartphone app feedback of average BPs after each session and could select charts from the app showing cumulative averages across weeks/months compared with threshold lines for BP control (ie, set at 140/90 mmHg). Reminders were sent every 3 days to the participants to prompt BP measurement adherence. If problems of BP measurement nonadherence or if the average of the last two BP readings per session was outside the threshold ranges, as specified by the practicing physician, the study coordinator would be alerted to contact the patient to obtain additional BP readings. If BP values persisted outside of threshold limits, the practicing physician's nurse manager would be alerted. This never occurred during the study.

The health care providers chose to receive bimonthly medication adherence and BP summary reports by email. These reports were tailored based upon their guidance to respond to the reporting needs of the providers (eg, synopsis of weekly adherence within 90 minutes of patient-designated times). After the 3-month trial evaluation was completed, MedMinders and smartphones were returned, with each patient receiving a home $\mathrm{BP}$ monitor.

\section{Feasibility measures}

Feasibility was assessed for SMASHers using retention rates, recruitment rates, medication adherence, BP adher- 
ence, and assessments from a health belief scale at the end of the 3 months. The provider was asked at the end of the trial if they would like to continue using the program for patient care. Medication adherence levels for the SMASH group from the MedMinder device were assessed using a modification of the Russell et al algorithm. ${ }^{33}$ Participants were instructed that in order to be fully compliant, all medications must be taken in a 3-hour window of the dosing time. Doses taken beyond 3 hours but within 6 hours (within 3 hours before or after the designated time) would receive a half score, and a missed dose was given a score of 0 . Daily scores ranged from 0 to 1 , with all daily scores averaged over the study length for adherence values.

BP adherence was assessed using the data received from the A\&D devices via the smartphone. BP adherence was measured by using total readings received divided by the total expected readings ( ix readings every 3 days). On-time BP adherence was measured by assessing the frequency with which participants measured BP at least 3 days after the last reading. This was calculated based on the following formula (100\% - [total number of time frames BP was monitored that were greater than 3 days/total possible number of 3-day time frames in the study]). Both adherence values had a maximum allowable score of $100 \%$ per individual. In addition, the number of times the interval was greater than 3 days was reported, including the maximum interval.

The health belief scale used four questions to assess the acceptability of the program. Questions were asked in Spanish and translate to: do you expect that the telehealth program would be effective in helping you to lower your blood pressure? Do you expect that the telehealth program would be effective in helping you to increase your consistency of taking your medication? Do you expect that the telehealth program would be effective in improving your overall health? Do you expect that the telehealth program would improve your general well-being? Score ranged on a 0-4 Likert scale with the responses: 0 , not at all; 1 , a little; 2, somewhat; 3 , a lot; 4 , very much.

\section{Statistical analyses}

Primary statistical analyses were conducted for feasibility measures, including statistics involving medication adherence data, BP adherence data, and results from the health belief scale for the SMASH group using averages and standard deviations. Recruitment and retention rates were also measured. Demographics were described using means and standard deviations, including calculation of body mass index $\left(\mathrm{kg} / \mathrm{m}^{2}\right)$. Secondary outcomes for resting and ambulatory BP between the SMASH and standard care groups assessed longitudinal changes both within and between each group. These were examined at pre-intervention, and months 1, 2, and 3 using independent-samples Mann-Whitney $U$ nonparametric tests to examine between-group differences, while Wilcoxon signed-rank tests were used to compare changes between pre-intervention and successive intervention months. Statistical significance was set at $P=0.05$.

\section{Results}

Twelve of 14 patients (86\%) approached who met the initial inclusion criteria consented to participate in the BP screening. The two who did not consent stated that they were not interested in sharing their personal information or address. Ten of these 12 patients subsequently showed uncontrolled systolic essential hypertension during the screening process and all consented to participate in the trial. After randomization, all ten completed the 3-month trial protocol. Table 2 shows that pre-intervention demographics and anthropometrics were comparable between the two groups, with the SMASH group having a higher body mass index on average. Medications were taken in the morning, at night, or at both times.

\section{Feasibility}

Feasibility of the program was shown in all the measures of participant acceptance. Both the SMASH and standard care groups had $100 \%$ retention rates throughout the 3 -month trial. For medication adherence, the SMASH participants demonstrated high overall protocol acceptability with monthly and overall percentages (month 1, 98.6\% $\pm 1.9 \%$; month 2, 96.8\% $\pm 5.4 \%$; month 3, 96.3\% $\pm 7.0 \%$; overall, 97.2\% $\pm 2.8 \%$ ). On-time BP adherence values showed that participants measured their BP

Table 2 Individual and group baseline descriptive and anthropometric characteristics

\begin{tabular}{lllll}
\hline Groups & Age (years) & Sex & BMI $\left(\mathbf{k g} / \mathbf{m}^{2}\right)$ & Waist $(\mathbf{c m})$ \\
\hline SMASH & & & & \\
I & 25 & Male & 46.3 & 135 \\
2 & 52 & Female & 30.8 & 95 \\
3 & $5 \mathrm{I}$ & Male & 32.4 & 112 \\
4 & 44 & Female & 57.1 & 150 \\
5 & $4 \mathrm{I}$ & Female & 23.8 & 75 \\
Mean (SD) & $42.6(10.9)$ & & $38.1(13.4)$ & $113.4(30.1)$ \\
SC & & & & \\
I & 24 & Male & 26.6 & 91 \\
2 & 46 & Male & 43.6 & 135 \\
3 & 54 & Female & 25.3 & 91 \\
4 & 40 & Female & 25.3 & 81 \\
5 & 39 & Male & 34.5 & 116 \\
Mean (SD) & $40.6(\mid 1.0)$ & & $31.1(8.0)$ & $102.8(22.2)$ \\
\hline
\end{tabular}

Abbreviations: SC, standard care; SD, standard deviation; BMI, body mass index; waist, waist circumference; SMASH, Smartphone Medication Adherence Stops Hypertension. 
every 3 days $83.2 \% \pm 6.0 \%$ of the time. Overall BP adherence for the total of expected readings was $89.2 \% \pm 19.06 \%$. On average, SMASH participants had $5 \pm 1.8$ episodes where subsequent $\mathrm{BP}$ readings were greater than 3 days, with a maximum interval of $8.6 \pm 4.0$ days. Results from the health benefits scale (scored 0-4) resulted in scores of 3.6 or higher on all items. All SMASH participants chose the highest rating (4) for the first three questions pertaining to the program being effective to help lower their BP, helping them increase taking their medications, and improving their overall health. The fourth question on improving general well-being averaged 3.6, with only one person not scoring the highest rating.

There were two providers in this study serving as primary care physicians for the SMASH participants. In routine clinical operations, the providers used a clinic nurse coordinator to review the medication adherence and BP reports. The providers showed acceptance of the program and used the summary reports to titrate medications when needed. One patient in the SMASH group had their dosages titrated (ie, dosage change) twice during the first month. For the first change, the physician mandated that the patient make a separate clinic visit to verify that their BP was elevated. The second medication change was made remotely based upon home BP data. Medication updates were made by the research technician in the MedMinder file. No medications were changed in the standard care group. Providers requested to keep using the SMASH system and indicated that it would be useful with other patients.

\section{Resting BP}

Resting BP showed a marked drop in the SMASH group from pre-intervention across the 3 intervention months. For example, by the third month, the SMASH group's systolic BP had declined by $47.2 \mathrm{mmHg}$ compared with $12 \mathrm{mmHg}$ in the standard care group. Mann-Whitney $U$ tests showed betweengroup differences at months $2(P=0.016)$ and $3(P=0.008)$, which are shown in Figure 2. The SMASH group showed significant decreases in BP from pre-intervention values to months 1,2 , and 3 (all $P=0.043$ ). The standard care group also exhibited a significant change from pre-intervention

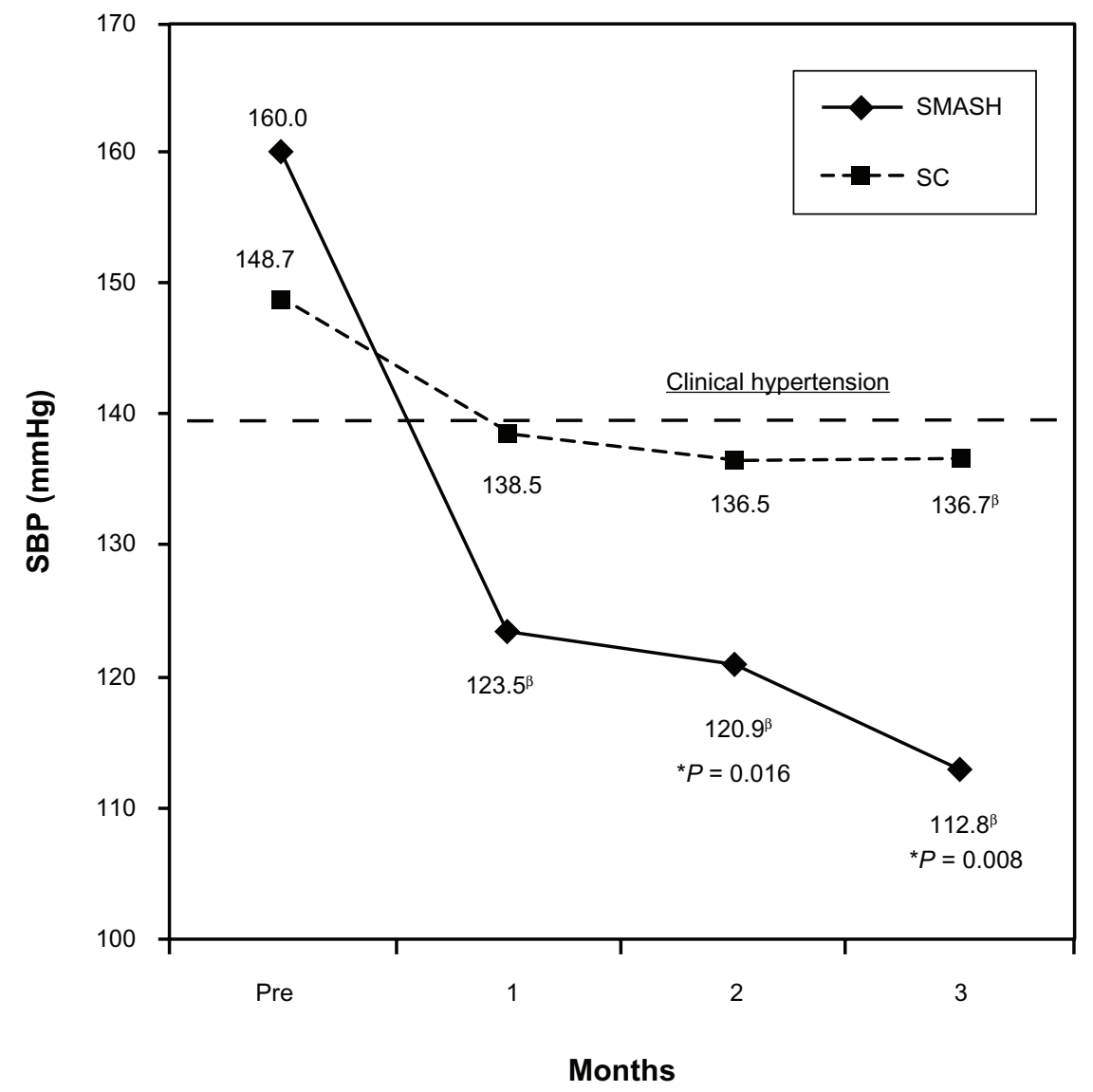

Figure 2 Resting SBP in Hispanic patients with essential hypertension allocated to SC $(n=5)$ and SMASH $(n=5)$ groups.

Notes: Nonparametric tests using independent-samples *Mann-Whitney $U$ tests for between groups and $\beta$ Wilcoxon signed-rank tests to examine differences within groups for changes from pre-intervention. Significance was set at $P=0.05$.

Abbreviations: SBP, systolic blood pressure; SC, standard care; SMASH, Smartphone Medication Adherence Stops Hypertension. 
to month $3(P=0.043)$. Decreases in systolic BP in the standard care group were due to one patient initiating and maintaining medication adherence based upon patient selfreport. The other standard care participants did not show BP declines. All but one participant from the SMASH group had resting systolic BP values (range $111-125 \mathrm{mmHg}$ ) below the $120 \mathrm{mmHg}$ threshold for JNC7 normal BP, whereas the standard care group had no participants below $120 \mathrm{mmHg}$ (range 128-144 $\mathrm{mmHg}$ ).

\section{Ambulatory BP}

Comparing ambulatory readings between the SMASH and standard care groups at each month showed large reductions in systolic BP for the SMASH group, even though statistical significance was not met using nonparametric statistics (all $P \geq 0.25$ ), see Figure 3. Additionally, statistical significance was not met for either group when comparing pre-intervention with subsequent follow-up months. Changes in scores (followup month - pre-intervention month) for SMASH and standard care groups for 24-hour readings showed differences of -14.3 versus -3.0 ( 1 month), -13.1 versus 4.8 ( 2 months), and -17.0 versus 3.5 (3 months), respectively. Differences between the SMASH and standard care groups for wake readings between pre-intervention and the follow-up months showed differences of -14.0 versus -1.8 ( 1 month), -9.3 versus 0.7 ( 2 months), and -15.6 versus 2.0 (3 months), respectively. Differences between the SMASH and standard care groups for sleep readings between pre-intervention and follow-up months showed differences of -14.1 versus -4.9 ( 1 month), -15.1 versus 5.5 ( 2 months), and -17.1 versus -0.1 (3 months), respectively.

\section{Discussion}

The current study involved an initial proof of concept evaluation of an mHealth medication and BP self-management system. We observed high acceptability via high study recruitment (86\%), retention (100\%), and good to excellent adherence to the mHealth program (average 97\% adherence to medication regimen across trial, $83 \%$ adherence to 3-day BP protocol, $89 \%$ to total BP readings). SMASH participants felt that the program helped them take their medications and improved their health. Provider acceptance showed that the feedback reports were helpful in modifying patients' medications quicker than would otherwise occur with clinic visits typically 3-6 months apart. It is important in a feasibility study to assess whether the target population can be readily recruited, determine if the protocol is acceptable to the parties involved, and whether there are data to support potentially meaningful clinical findings if an efficacy pilot study that was adequately powered was instituted. Data showed supportive results in all these areas. Anecdotally, after trial completion, two SMASHers reported purchasing inexpensive pill boxes and used their own cell phones to remind them to take their

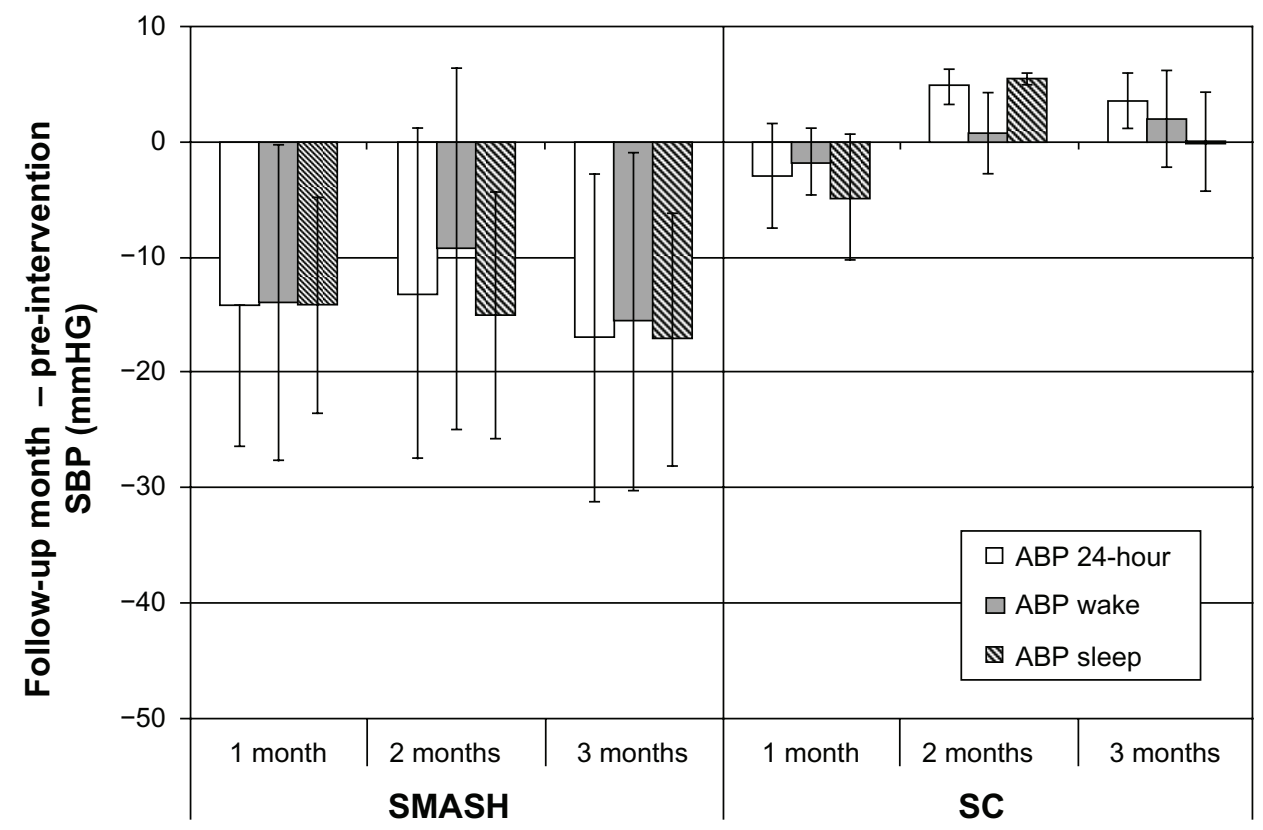

Figure 3 Overall change scores (intervention month - pre-intervention) for 24-hour, wake, and sleep ABP for SMASH $(n=5)$ and standard care $(n=5)$ groups. Error bars represent standard error of the mean. Wake represents readings between 8 am to 10 pm and sleep represents those between 12 am to 6 am. Differences compared between groups used independent-samples Mann-Whitney $U$ tests (all $P \geq 0.25$ ). Within-group differences in changes between pre-intervention to intervention months was analyzed using Wilcoxon signed-rank tests (all $P>0.1$ ).

Abbreviations: ABP, ambulatory blood pressure; SC, standard care; SMASH, Smartphone Medication Adherence Stops Hypertension; SBP, systolic blood pressure. 
medications. We believe these behaviors suggest that the two primary components of self-determination theory needed to sustain engagement in the desired behaviors were achieved, ie, high self-efficacy and autonomous regulation. ${ }^{30,31}$ Future RCTs will include self-report measures of these constructs to gauge how well the personalized motivational and reinforcement messages are impacting these constructs. These constructs may also support the notion of developing life skills and habits that may be reinforced by environmental triggers or cuing mechanisms. ${ }^{34}$ For example, having the MedMinder reminders may have created a daily cue that corresponded with a specific event in the patients' schedules, such as breakfast, brushing teeth, or other contexts that persisted after the device was taken away.

With regard to BP changes, meaningful clinical reductions in resting and ambulatory SBP were observed for the SMASH group whereas the standard care group showed little to no changes. In regard to clinic-based BP changes, we found that each SMASHer achieved JNC7-designated BP control by month 2 (ie, $<140 / 90 \mathrm{mmHg}$ in clinic) and maintained this control across the trial. ${ }^{1}$ Sustained control of BP levels in SMASHers was evident across the 3-month trial and coincided with their high adherence measures observed with mobile devices. Previous BP self-monitoring trials have observed significant BP reductions, but the degree of reduction observed in the present study was larger compared with previous studies (eg, systolic BP at 3 months was $-47.2 \mathrm{mmHg}$ versus an average of $-8 \mathrm{mmHg}$ across previous RCTs. ${ }^{5,18,19}$ The study by Lai which involved Hispanic hypertensives using face-to-face consultation sessions with their pharmacists was successful in reducing their BP levels to the stage 2 pre-hypertensive range (ie, 130-139/85-89 mmHg) in their 6-month trial. ${ }^{21}$ The SMASH system further reduced Hispanics' BP to stage 1 pre-hypertension (121-129/81-84 mmHg) or optimal normotensive levels (ie, $<120 / 80 \mathrm{mmHg}$ ). Collectively, the large degree of sustained BP reductions observed is quite remarkable given the relative simplicity and low contact method of the mHealth program compared with multimodal face-to-face educational and cognitive behavioral skills-based approaches used in previous RCTs. ${ }^{5,16,18,19}$ Although these improvements in BP control are promising and show some indications that meaningful changes in BP control were observed, they should be interpreted cautiously due to the limited sample size. It is possible that although the groups were randomized, the intervention group may have included all highly motivated individuals, although we have previously found high acceptability when using mHealth technology in Hispanic groups. ${ }^{22}$ A future properly powered efficacy trial will help to address such potential issues and determine whether effects are replicated across a longer time period.

The primary purpose of this study was to test a patientcentered mHealth medication and BP self-management program in Hispanic hypertensives and determine its acceptability and possible clinical impact. Even though our limited sample size should be considered carefully when interpreting the results of the study, our preliminary results show great potential to enhance medication adherence rates and improve BP outcomes. The high level of success of the SMASH mHealth program in reaching and sustaining JNC 7-designated BP control among previously uncontrolled hypertensives may be partly attributable to our utilization of an iterative design process guided by patient and health care provider feedback and suggestions using behavioral change theory and technology acceptance model tenets. ${ }^{30,35} \mathrm{~A}$ critical component to the success of any mHealth program is the willingness and ability of the target population and providers to adopt and effectively utilize the technology. In addition, program design should be based upon theoretical behavioral change principles shown to be effective in sustaining the desired behaviors. ${ }^{36-38}$ Following these principles, we initially conducted key informant interviews with Hispanic hypertensives and health care providers, followed by formal survey evaluations to determine Hispanics' use of mobile technology, attitudes toward mHealth, and development of SMASH prototype designs. ${ }^{22}$ These concepts have been useful in our team's development and implementation for both face-to-face and mHealth-delivered cognitive behavioral change programs, resulting in sustained behavior changes and improvements in associated biofunction indices. ${ }^{28,39-41}$ This repeated contact with Hispanic patients and their health care teams enabled us to develop and refine our approach to implement a personalized program aimed at initiating and sustaining motivation to adhere to medical regimens by improving self-efficacy and autonomous regulation. ${ }^{30}$ Physicians determined when their clinic nurse coordinator should be immediately contacted if and when a patient's BP exceeded threshold levels. They also determined the content and delivery format for patients' summary reports to help ensure timely medication adjustments. This iterative focus enabled each component of the SMASH system to be developed, modified, optimized, and refined before the SMASH system was formally evaluated in the proof of concept RCT.

Principles of patient-centered care highlight partnering with intended users and implementers are important when developing a culturally sensitive intervention that is 
meaningful and effective. Our research involving ethnic minorities has found that cultural heritage, attitudes, beliefs of treatment efficacy, and buy-in play significant roles in the degree to which an intervention is adopted..$^{28,29,39,41}$ This information has and will continue to guide the iterative design, evaluation, and refinement process of the SMASH program. With newly capable data management systems in place to automate personalized messages, this intervention can be scaled to a large population. Next steps will include a small-scale efficacy RCT followed by further refinement and a subsequent multisite efficacy/effectiveness RCT. Ultimately, the goal is to reach large-scale dissemination and utilizing programs such as this for best practice health care models in self-management of essential hypertension.

\section{Acknowledgments}

Verizon Wireless of South Carolina provided use of the Motorola Droid smartphones. This paper was supported by a pilot grant from the South Carolina Clinical and Translational Research Institute, with an academic home at the Medical University of South Carolina, CTSA National Institutes of Health/National Center for Research Resources (UL1RR029882 and UL1 TR000062), Duke Endowment Award 6355-SP, and a Verizon Foundation Award. The contents are solely the responsibility of the authors and do not necessarily represent the official views of the National Institutes of Health or National Center for Research Resources.

\section{Disclosure}

The authors report no conflicts of interest in this work.

\section{References}

1. Chobanian AV, Bakris GL, Black HR, et al. The Seventh Report of the Joint National Committee on Prevention, Detection, Evaluation, and Treatment of High Blood Pressure: the JNC 7 report. JAMA. 2003;289(19):2560-2572.

2. Egan BM, Zhao Y, Axon RN. US trends in prevalence, awareness, treatment, and control of hypertension, 1988-2008. JAMA. 2010;303(20): 2043-2050.

3. Humes KR, Jones NA, Ramirez RR, United States' Bureau of the Census. Overview of race and Hispanic origin, 2010. Washington, DC: US Deptartment of Commerce, Economics and Statistics Administration, US Census Bureau; 2011.

4. Ostchega Y, Yoon SS, Hughes J, Louis T. Hypertension awareness, treatment, and control - continued disparities in adults: United States, 2005-2006. NCHS Data Brief. 2008;(3):1-8.

5. Agarwal R, Bills JE, Hecht TJ, Light RP. Role of home blood pressure monitoring in overcoming therapeutic inertia and improving hypertension control: a systematic review and meta-analysis. Hypertension. 2011;57(1):29-38.

6. Dusing R. Overcoming barriers to effective blood pressure control in patients with hypertension. Curr Med Res Opin. 2006;22(8): 1545-1553.
7. Osterberg L, Blaschke T. Adherence to medication. $N$ Engl $J$ Med. 2005;353(5):487-497.

8. Okonofua EC, Simpson KN, Jesri A, Rehman SU, Durkalski VL, Egan BM. Therapeutic inertia is an impediment to achieving the Healthy People 2010 blood pressure control goals. Hypertension. 2006;47(3): 345-351.

9. Ho PM, Bryson CL, Rumsfeld JS. Medication adherence: its importance in cardiovascular outcomes. Circulation. 2009;119(23):3028-3035.

10. Cramer JA, Benedict A, Muszbek N, Keskinaslan A, Khan ZM. The significance of compliance and persistence in the treatment of diabetes, hypertension and dyslipidaemia: a review. Int J Clin Pract. 2008;62(1): 76-87.

11. Romanelli RJ, Schiro TA, Jukes T, Wong KS, Ishisaka DY. Disparities in blood pressure control within a community-based provider network: an exploratory analysis. Ann Pharmacother. 2011;45(12):1473-1482.

12. Bramley TJ, Gerbino PP, Nightengale BS, Frech-Tamas F. Relationship of blood pressure control to adherence with antihypertensive monotherapy in 13 managed care organizations. J Manag Care Pharm. 2006;12(3):239-245.

13. Esposti LD, Di Martino M, Saragoni S, et al. Pharmacoeconomics of antihypertensive drug treatment: an analysis of how long patients remain on various antihypertensive therapies. $J$ Clin Hypertens (Greenwich). 2004;6(2):76-84.

14. Perez A. Self-management of hypertension in Hispanic adults. Clin Nurs Res. 2011;20(4):347-365.

15. Ventura H, Pina IL, Lavie CJ. Hypertension and antihypertensive therapy in Hispanics and Mexican Americans living in the United States. Postgrad Med. 2011;123(6):46-57.

16. Carter BL, Bergus GR, Dawson JD, et al. A cluster randomized trial to evaluate physician/pharmacist collaboration to improve blood pressure control. J Clin Hypertens (Greenwich). 2008;10(4):260-271.

17. Von Muenster SJ, Carter BL, Weber CA, et al. Description of pharmacist interventions during physician-pharmacist co-management of hypertension. Pharm World Sci. 2008;30(1):128-135.

18. Glynn LG, Murphy AW, Smith SM, Schroeder K, Fahey T. Interventions used to improve control of blood pressure in patients with hypertension. Cochrane Database Syst Rev. 2010;3:CD005182.

19. Cappuccio FP, Kerry SM, Forbes L, Donald A. Blood pressure control by home monitoring: meta-analysis of randomised trials. $B M J$. 2004;329(7458): 145 .

20. Logan AG, McIsaac WJ, Tisler A, et al. Mobile phone-based remote patient monitoring system for management of hypertension in diabetic patients. Am J Hypertens. 2007;20(9):942-948.

21. Lai LL. Community pharmacy-based hypertension disease-management program in a Latino/Hispanic-American population. Consult Pharm. 2007;22(5):411-416.

22. Price M, Williamson D, McCandless R, et al. Hispanic migrant farm workers' attitudes toward mobile phone-based telehealth for management of chronic health conditions. $J$ Med Internet Res. 2013;15(4):e76

23. Nielsen Mobile Insights. America's new mobile majority: a look at smartphone owners in the US. Newswire 2012. Available from: http:// www.nielsen.com/us/en/newswire/2012/who-owns-smartphones-inthe-us.html. Accessed August 16, 2013.

24. Reinders A, Reggiori F, Shennan AH. Validation of the DINAMAP ProCare blood pressure device according to the international protocol in an adult population. Blood Press Monit. 2006;11(5):293-296.

25. Li Z, Snieder H, Harshfield GA, Treiber FA, Wang X. A 15-year longitudinal study on ambulatory blood pressure tracking from childhood to early adulthood. Hypertens Res. 2009;32(5): 404-410.

26. Wang X, Ding X, Su S, Harshfield G, Treiber F, Snieder H. Genetic influence on blood pressure measured in the office, under laboratory stress and during real life. Hypertens Res. 2011;34(2):239-244.

27. O'Brien E, Mee F, Atkins N, O'Malley K. Accuracy of the SpaceLabs 90207 determined by the British Hypertension Society protocol. J Hypertens. 1991;9(6):573-574. 
28. Gregoski MJ, Barnes VA, Tingen MS, Dong Y, Zhu H, Treiber FA. Differential impact of stress reduction programs upon ambulatory blood pressure among African American adolescents: influences of endothelin-1 gene and chronic stress exposure. Int J Hypertens. 2012;2012:510291.

29. Gregoski MJ, Barnes VA, Tingen MS, Harshfield GA, Treiber FA. Breathing awareness meditation and LifeSkills Training programs influence upon ambulatory blood pressure and sodium excretion among African American adolescents. J Adolesc Health. 2011;48(1):59-64.

30. Ryan RM, Deci EL. Self-determination theory and the facilitation of intrinsic motivation, social development, and well-being. Am Psychol. 2000;55(1):68-78.

31. Ryan R, Patrick H, Deci E, Williams G. Facilitating health behaviour change and its maintenance: Interventions based on self-determination theory. Available from: http://www.selfdeterminationtheory.org/SDT/ documents/2008_RyanPatrickDeciWilliams_EHP.pdf. Accessed August 16, 2013.

32. Verdecchia P, Angeli F, Poeta F, et al. Validation of the A\&D UA-774 (UA-767Plus) device for self-measurement of blood pressure. Blood Press Monit. 2004;9(4):225-229.

33. Russell C, Conn V, Ashbaugh C, et al. Taking immunosuppressive medications effectively (TIMELink): a pilot randomized controlled trial in adult kidney transplant recipients. Clin Transplant. 2010;25(6): 864-870.

34. Wood W, Neal DT. A new look at habits and the habit-goal interface. Psychol Rev. 2007;114(4):843-863.
35. Legris $\mathrm{P}$, Ingham $\mathrm{J}$, Collerette $\mathrm{P}$. Why do people use information technology? A critical review of the technology acceptance model. Inform Manage. 2003;40(3):191-204.

36. Collins LM, Murphy SA, Nair VN, Strecher VJ. A strategy for optimizing and evaluating behavioral interventions. Ann Behav Med. 2005;30(1):65-73.

37. Craig P, Dieppe P, Macintyre S, Michie S, Nazareth I, Petticrew M. Developing and evaluating complex interventions: the new Medical Research Council guidance. BMJ. 2008;337:a1655.

38. Riley WT, Rivera DE, Atienza AA, Nilsen W, Allison SM, Mermelstein R. Health behavior models in the age of mobile interventions: are our theories up to the task? Transl Behav Med. 2011;1(1):53-71.

39. Barnes VA, Gregoski MJ, Tingen MS, Treiber FA. Influences of family environment and meditation efficacy on hemodynamic function among African American adolescents. J Complement Integr Med. 2010;7(1):1326.

40. Gregoski M, Mueller M, Jackson B, Frenzel R, Sprehn S, Treiber F. Development and validation of a smart phone heart rate acquisition application for health promotion and wellness telehealth applications. Int J Telemed Appl. 2012;2012:696324.

41. Gregoski MJ, Mueller M, Jackson B, Vertegel A, Sharporev A, Treiber F. Tension tamer: delivering meditation with objective heart rate acquisition for adherence monitoring using a smart phone platform. JAltern Complement Med. 2013;19(1):17-19.
Smart Homecare Technology and TeleHealth

\section{Publish your work in this journal}

Smart Homecare Technology and TeleHealth is an international, peer-reviewed, open access online journal publishing original research, reviews, editorials and commentaries on the application of technology to support people and patients at home and in assisted living centers to optimize healthcare and management resources. Specific topics in the journal include: Development and application of

\section{Dovepress}

devices within the home and embedded in appliances; Healthcare provider communication and education tools; and drug ordering and adherence. The manuscript management system is completely online and includes a very quick and fair peer-review system, which is all easy to use. Visit http://www.dovepress.com/ testimonials.php to read real quotes from published authors. 